Jis Thomas*, Tawa Olayemi Olukade, Aliya Naz, Husam Salama, Mai Al-Qubaisi, Hilal Al Rifai and Sawsan Al-Obaidly

\title{
The neonatal respiratory morbidity associated with early term caesarean section - an emerging pandemic
}

https://doi.org/10.1515/jpm-2020-0402

Received August 21, 2020; accepted April 22, 2021;

published online May 7, 2021

\section{Abstract}

Objectives: To examine the impact of early term caesarean section (CS) on respiratory morbidity and early neonatal outcomes when elective caesarean section was carried out before 39 completed weeks gestation in our population.

Methods: A one-year population-based retrospective cohort analysis using routinely collected hospital data. Livebirths from women who had elective lower segment cesarean section (ELSCS) for uncomplicated singleton pregnancies at early term (ET) $37+0$ to $38+6$ weeks were compared to full term $(\mathrm{FT}) \geq 39+0$ weeks gestation. Exclusion criteria included diabetes, antenatal corticosteroid use, stillbirths, immediate neonatal deaths, normal vaginal deliveries and emergency caesareans sections. The outcomes were combined respiratory morbidity (tachypnea [TTN] and respiratory distress syndrome [RDS]), Apgar $<7$ at 5 min of age, respiratory support, duration of respiratory support and NICU admission.

Results: Out of a total of 1,466 elective CS with term livebirths, the timing of CS was early term (ET) $n=758$ (52\%) and full term (FT) $n=708$ (48\%). There was a higher incidence of respiratory morbidities and neonatal outcomes in

*Corresponding author: Dr. Jis Thomas, Department of Obstetrics and Gynecology, Women's Wellness and Research Centre, Hamad Medical Corporation, Doha, Qatar; and Royal College of Obstetricians and Gynecologists, PO Box: 3050, Doha, Qatar, Phone: +0097455309179, E-mail: jthomas17@hamad.qa

Tawa Olayemi Olukade, Husam Salama, Mai Al-Qubaisi and Hilal Al Rifai, Neonatal Intensive Care Unit, Women's Wellness and Research Center, Doha, Qatar

Aliya Naz, Royal College of Obstetricians and Gynecologists, Doha, Qatar; Weill Cornell Medicine, Doha, Qatar; and Sidra Medical and Research Centre, Doha, Qatar

Sawsan Al-Obaidly, Department of Obstetrics and Gynecology, Women's Wellness and Research Centre, Hamad Medical Corporation, Doha, Qatar; and Weill Cornell Medicine, Doha, Qatar the ET in comparison to FT newborns. In the univariable analysis, significant risks for outcomes were: the need for oxygen support OR 2.42 (95\% C.I. 1.38-4.22), respiratory distress syndrome and/or transient tachypnea of newborn (RDSF/TTN) OR 2.44 (95\% C.I. 1.33-4.47) and neonatal intensive care unit (NICU) admission OR 1.91 (95\% C.I. 1.22-2.98). Only the need for oxygen support remained (OR $1.81,95 \%$ C.I. 1.0-3.26) in the multivariable analysis. These results were observed within the context of a significantly higher proportion of older, multiparous, and higher number of previous caesarean sections in the early term CS group.

Conclusions: There is a significant risk of respiratory morbidities in infants born by elective cesarean section prior to full term gestation. Obstetricians should aim towards reducing the high rate of women with previous multiple cesarean sections including balancing the obstetric indication of early delivery among such women with the evident risk of neonatal respiratory morbidity.

Keywords: early term caesarean section; elective lower segment caesarean section; full term caesarean section; neonatal respiratory morbidity; NICU admission.

\section{Introduction}

There is an alarming and consistent global rise in the rates of cesarean sections accounting for almost 21 percent of the total global birth [1, 2]. Despite being a lifesaving procedure in medically indicated cases, the unprecedented rise in caesarean rates is disturbing as is associated with significant short term and long term maternal and neonatal morbidities [3]. Published literature reports that when compared with vaginal birth, elective caesarean birth is associated with a two to almost seven fold increase in the risk of respiratory morbidities (in terms of transient tachypnea of newborn [TTN], respiratory distress syndrome [RDS], persistent pulmonary hypertension and neonatal intensive care unit [NICU] admission) in the near term neonate $[4,5]$. There is consistent evidence from prospective large multicenter studies that the magnitude of 
this morbidity is augmented when cesarean section is performed before 39 completed weeks gestation [6, 7]. Therefore, many professional international organizations recommend that elective $\mathrm{CS}$ should not be routinely carried out before 39 weeks in uncomplicated singleton pregnancies [8-10]. Despite this, a huge number of caesarean section (CS) are being performed worldwide, for various reasons before 39 completed weeks. The neonatal outcomes of early term CS (between $37+0-38+6$ weeks) is therefore a subject of thorough research in many parts of the world.

Even though our national guideline recommends delaying elective lower caesarean section (ELCS) in uncomplicated singleton pregnancy up to 39 completed weeks, we are unable to completely adhere to this policy due to various factors. On many occasions, the rationale stated behind the non-compliance to guidelines is, prioritization of maternal interests over the fetal well-being. Nevertheless, recent literature suggests that $10 \%$ of women booked for caesarean section at 39 weeks will go into labour prior to the scheduled date. Therefore, obstetricians tend to schedule elective caesarean sections prior to 39 completed weeks. Intrapartum caesarean sections significantly increase serious maternal and neonatal morbidities. In addition, limitation of resources also plays a major role in non-adherence to the guidelines. The magnitude of neonatal respiratory morbidity due to noncompliance to the national guideline in our population, has not been investigated previously. We therefore aimed to examine the impact of early term CS on respiratory morbidity and early neonatal outcomes in the neonate when elective caesarean section is carried out before 39 completed weeks in our population.

\section{Materials and methods}

\section{Study design}

This was a population-based retrospective cohort analysis from the PEARL-Peristat registry. The PEARL-Peristat Study (Perinatal Neonatal Outcomes Research Study in the Arabian Gulf) is an ongoing population-based study of mainly routinely collected hospital data for mother-child birth cohorts in Qatar. The study is to study immediate and long term foeto-maternal pregnancy outcomes. Data collection started in January 2017 with a planned sample size of 35,000 births nationwide. The study is funded by Qatar National Research Fund (QNRF) and sponsored by the Medical Research Centre, Hamad Medical Corporation. The study was approved by the Hamad Medical Corporation Institutional Review Board, with a waiver of written consent.

\section{Setting and participants}

Sample for this study included births conducted in Women's Wellness and Research Center, Qatar. This hospital accounts for the largest proportion of births conducted in the country. The hospital caters for more than $80 \%$ of the pregnant population in the country. All singleton births at $37+0$ weeks gestation and above between January and December 2017 were eligible for inclusion in this study. Exclusion criteria included diabetes in pregnancy, antenatal corticosteroid (betamethasone) use in pregnancy, stillbirths, immediate neonatal deaths in the delivery room/theatre, normal vaginal deliveries and emergency caesareans sections. Out of 14,787 term singletons births at 37 weeks gestation and above exported from registry, 4,295 births were excluded leaving a total of 10,492 births which included 1,466 elective caesarean births included in this analysis.

\section{Outcome variables}

The immediate neonatal outcomes studied included respiratory morbidity assessed by a $5 \mathrm{~min}$ Apgar score less than seven, respiratory distress syndrome or transient tachypnea of the newborn (RDS/TTN) termed combined respiratory morbidity, the need for oxygenation and the duration of respiratory support. Other outcomes included admission to neonatal intensive care unit and the duration of hospital stay. The need for respiratory support was also examined as none, noninvasive and invasive. In our setting, the diagnosis of transient TTN is based on clinical and radiologic findings characterized by an early onset of respiratory symptoms including tachypnea or in some cases, retractions, grunting, or nasal flaring with good response to oxygen supplementation [11]. Chest radiographic findings are often consistent with lung congestion (ibid). The symptoms and findings are usually transient and self-limiting, disappearing within the few days of life. Respiratory distress syndrome (RDS) is a syndrome in predominantly premature infants caused by developmental insufficiency of surfactant production and immaturity in the lungs [12]. It is characterized by symptoms of respiratory distress including hypoxemia and hypercapnia with a typical chest radiograph showing low lung volumes and a bilateral, reticular granular pattern (ground glass appearance) with air bronchograms.

\section{Covariates}

Maternal: Maternal age at delivery was categorised into $<35$ years and $\geq 35$ years, parity into nulliparous, $1-4$ or $\geq 5$ parous experiences and Nationality into Qatari vs. non-Qatari. The history of previous CS delivery was coded as yes/no and the number of previous CS into 0 (none), 1 (one), $2-3$ and $\geq 4$.

Newborn: Gestational age at delivery (GA) was grouped into $37+0-38+6$ weeks and $\geq 39+0$ weeks and gender as male or female.

\section{Statistical analysis}

We compared ELCS livebirths at $37+0-38+6$ weeks to births at $\geq 39+0$ weeks. Descriptive statistics was used to show the distribution of variables using numbers and percentages for categorical data, means 
and standard deviation or median and interquartile ranges for continuous data. The association between covariates, outcomes and gestational age were tested using Chi-square test or Fishers test and t-test or Mann Whitney U as appropriate. Univariable and multivariable logistic regression was performed to examine and test the association between neonatal outcomes and gestational age while adjusting for the effects of significant covariates. Statistical significance was set at $95 \%$ confidence intervals (CI) and $\mathrm{p}<0.05$. Statistical analysis was performed using IBM SPSS 22 statistical software.

\section{Results}

Overall, the 10,492 singleton livebirths of uncomplicated term pregnancies examined for inclusion in this study comprised of 7,795 (74.3\%) vaginal deliveries, 1,466 (14\%) ELCS, and 1,231 (11.7\%) emergency caesarean sections (EMCS). Of the 1,466 ELCS, early term (ET) CS were $52 \%$ (758/1,466), while full term (FT) were 48\% $(708 / 1,466)$. Maternal characteristics are as shown in Table 1. There were significantly more older women (35 years and above), multiparous, and Qatari women in the ET group. The ET group also had a significantly higher proportion of a history of previous CS and higher number of previous CS.

\section{Timing of elective caesarean section and neonatal morbidities}

Early term CS was significantly associated with more immediate adverse newborn outcomes. The rate of NICU admissions was 8 vs. 4.4\% in ET vs. FT babies (Table 2). An ET newborn had a significantly higher risk of getting admitted to NICU in comparison to a FT newborn OR 1.91 (95\% C.I. 1.22-2.98) (Table 3). This association was attenuated when the risk was adjusted for maternal age, parity, number of previous CS and maternal nationality aOR 1.48 (95\% C.I. 0.92-2.37). Further examination based on gestational age breakdown showed an inverse relationship between GA and NICU admission (17.2\% at 37 weeks, $5.1 \%$ at 38 weeks, $4.4 \%$ at $\geq 39$ weeks).

There were two babies with an Apgar score $<7$ at $5 \mathrm{~min}$ in the ET group and none in the FT group. While the overall incidence of combined respiratory morbidity (TTN/RDS) was $3.6 \%$ among all ELCS, it was 5 vs. $2.1 \%$ in ET vs. FT respectively (Table 2). The incidence in each age group was: $11.8 \%$ for 37 weeks, $2.8 \%$ for 38 weeks and $2.1 \%$ for 39 weeks of gestation, respectively. In the univariable analysis, ET babies were more than twice likely to have RDS/TTN, but this association was not significant when adjustment was made for other potential confounding variables aOR 1.67 (95\% C.I. 0.87-3.18).

Majority of the babies, about $97 \%$ did not require any form of respiratory support (Table 2). This notwithstanding, ET newborns were twice more likely to require respiratory support than FT newborns OR 2.42 (95\% C.I. 1.38-4.22). This need was still significant even after adjustment for other variables, aOR 1.81 (95\% C.I. 1.00-3.26) (Table 3). There were no association between timing of delivery, duration of respiratory support and hospital stay between the two groups.

\section{Timing of delivery, number of previous caesarean sections and neonatal morbidities}

There were significantly more women with a history of two or more previous CS in the ET group compared to the FT

Table 1: Maternal characteristics of 1,466 women with term elective caesarean section.

\begin{tabular}{|c|c|c|c|c|c|c|c|}
\hline & \multicolumn{7}{|c|}{ Gestational age at delivery } \\
\hline & \multicolumn{2}{|c|}{ Total, $n=1,466$} & \multicolumn{2}{|c|}{$37^{+0}-38^{+6}$ weeks, $n=758$} & \multicolumn{2}{|c|}{$\geq 39$ weeks, $n=708$} & \multirow[t]{2}{*}{$x^{2}, \mathrm{p}$-value } \\
\hline & $\mathbf{n}$ & $\%$ & $\mathbf{n}$ & $\%$ & $\mathbf{n}$ & $\%$ & \\
\hline Maternal age $\geq 35$ years & 382 & $26.1 \%$ & 239 & $31.5 \%$ & 143 & $20.2 \%$ & $<0.001$ \\
\hline Qatari & 469 & $32.0 \%$ & 274 & $36.1 \%$ & 195 & $27.5 \%$ & $<0.001$ \\
\hline \multicolumn{8}{|l|}{ Parity } \\
\hline Nulliparous & 138 & $9.4 \%$ & 54 & $7.1 \%$ & 84 & $11.9 \%$ & \\
\hline Para 1-4 & 1,229 & $83.8 \%$ & 634 & $83.6 \%$ & 595 & $84.0 \%$ & $<0.001$ \\
\hline Para $\geq 5$ & 99 & $6.8 \%$ & 70 & $9.2 \%$ & 29 & $4.1 \%$ & \\
\hline Previous CS & 1,194 & $81.4 \%$ & 635 & $83.8 \%$ & 559 & $79.0 \%$ & 0.018 \\
\hline \multicolumn{8}{|l|}{ No. of previous CS } \\
\hline 0 & 272 & $18.6 \%$ & 123 & $16.2 \%$ & 149 & $21.0 \%$ & $<0.001$ \\
\hline 1 & 572 & $39.0 \%$ & 210 & $27.7 \%$ & 362 & $51.1 \%$ & \\
\hline $2-3$ & 550 & $37.5 \%$ & 360 & $47.5 \%$ & 190 & $26.8 \%$ & \\
\hline$\geq 4$ & 72 & $4.9 \%$ & 65 & $8.6 \%$ & 7 & $1.0 \%$ & \\
\hline
\end{tabular}


Table 2: Pregnancy outcomes of 1,466 women with term elective caesarean sections.

\begin{tabular}{|c|c|c|c|c|c|c|c|}
\hline & \multicolumn{2}{|c|}{ Total, $n=1,466$} & \multicolumn{2}{|c|}{$\begin{array}{c}37^{+0}-38^{+6} \\
\text { weeks, } n=758\end{array}$} & \multicolumn{2}{|c|}{$\begin{array}{c}\geq 39 \text { weeks, } \\
n=708\end{array}$} & \multirow[t]{2}{*}{$x^{2}, p$-value } \\
\hline & $\mathbf{n}$ & $\%$ & $\mathbf{n}$ & $\%$ & $\mathbf{n}$ & $\%$ & \\
\hline Gender, F & 721 & $49.2 \%$ & 360 & $47.5 \%$ & 361 & $51.0 \%$ & 0.181 \\
\hline Birth weight, mean \pm SD & \multicolumn{2}{|c|}{$3,269 \pm 444$} & \multicolumn{2}{|c|}{$3,166 \pm 426$} & \multicolumn{2}{|c|}{$3,380 \pm 437$} & $<0.001$ \\
\hline RDS/TTN & 53 & $3.6 \%$ & 38 & $5.0 \%$ & 15 & $2.1 \%$ & 0.003 \\
\hline Apgar at $5 \mathrm{~min}<7$ & 2 & $0.1 \%$ & 2 & $0.3 \%$ & 0 & $0.0 \%$ & NA \\
\hline \multicolumn{8}{|l|}{ Respiratory support } \\
\hline None & 1,403 & $95.7 \%$ & 713 & $94.1 \%$ & 690 & $97.5 \%$ & \\
\hline Non-invasive & 48 & $3.3 \%$ & 36 & $4.7 \%$ & 12 & $1.7 \%$ & \\
\hline $\mathrm{CMV} / \mathrm{HFV}$ & 15 & $1.0 \%$ & 9 & $1.2 \%$ & 6 & $0.8 \%$ & 0.004 \\
\hline Any oxygenation & 63 & $4.3 \%$ & 45 & $5.9 \%$ & 18 & $2.5 \%$ & 0.001 \\
\hline NICU admission & 92 & $6.3 \%$ & 61 & $8.0 \%$ & 31 & $4.4 \%$ & 0.004 \\
\hline \multicolumn{8}{|l|}{ Days, mean $\pm S D$} \\
\hline Baby hospital duration $^{\text {b }}$ & \multicolumn{2}{|c|}{$3.2 \pm 7.1$} & \multicolumn{2}{|c|}{$3.3 \pm 6.6$} & \multicolumn{2}{|c|}{$3.2 \pm 7.6$} & 0.702 \\
\hline Duration of respiratory support ${ }^{\mathrm{a}}$, median (IQR) & \multicolumn{2}{|c|}{$1.3(0.8-3.3)$} & \multicolumn{2}{|c|}{$1.3(0.8-3.3)$} & \multicolumn{2}{|c|}{$1.1(0.8-4.2)$} & 0.891 \\
\hline
\end{tabular}

${ }^{\mathrm{a}}$ Mann Whitney U test; ${ }^{\mathrm{b}}$ t-test; NA, not applicable.

Table 3: Association between gestational age at birth and neonatal morbidities in early term and late term elective caesarean sections.

\begin{tabular}{|c|c|c|c|c|}
\hline & \multicolumn{2}{|c|}{ Crude odds ratio } & \multicolumn{2}{|c|}{ Adjusted odds ratio } \\
\hline & OR & $(95 \% \mathrm{Cl})$ & OR & $(95 \% \mathrm{Cl})$ \\
\hline NICU admission & 1.91 & $1.22-2.98^{\mathrm{a}}$ & 1.48 & $0.92-2.37$ \\
\hline RDS/TTN & 2.44 & $1.33-4.47^{\mathrm{a}}$ & 1.67 & $0.87-3.18$ \\
\hline Any oxygenation & 2.42 & $1.38-4.22^{\mathrm{a}}$ & 1.81 & $1.00-3.26^{\mathrm{a}}$ \\
\hline
\end{tabular}

Adjusted for maternal age, parity, no. of previous CS (continuous) and

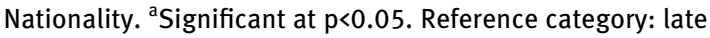
term $\geq 39+0$ weeks.

group and a higher number of previous CS was directly proportional to a higher incidence of the neonatal outcomes (Figure 1).

\section{Discussion}

In our study, we aimed to evaluate the impact of early term CS on early neonatal outcomes and respiratory morbidity in our population. Out of a total of 1,466 patients with uncomplicated singleton pregnancies who underwent elective CS, our findings showed that neonatal respiratory morbidity and outcomes were determined largely by the gestational age at delivery. Combined neonatal respiratory morbidity (TTN/RDS) was inversely related to the gestational age at delivery with the lowest incidence observed when caesarean section was performed at 39 weeks. Likewise, the neonates requiring oxygenation, invasive and noninvasive ventilation declined when elective caesarean
Number of previous CS and RDS/TTN

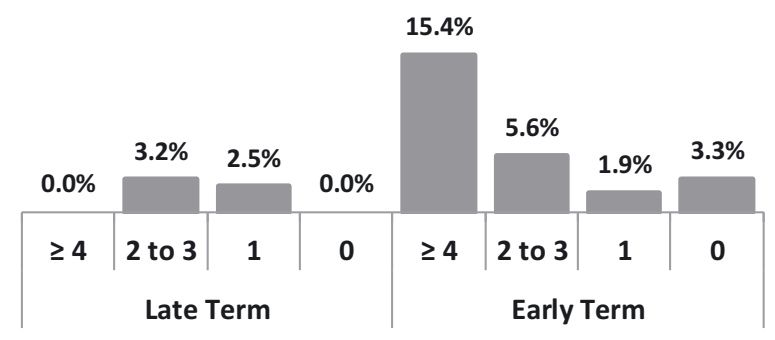

Number of previous CS and NICU admission

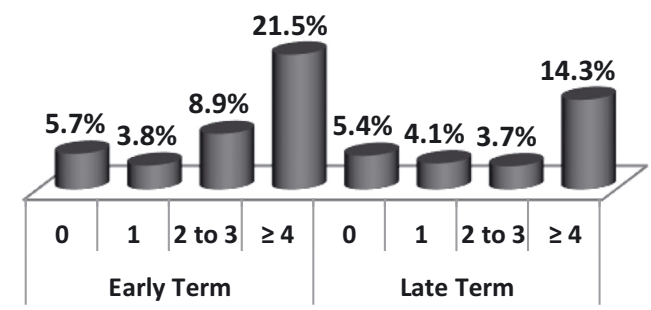

Number of previous CS and need for Oxygenation

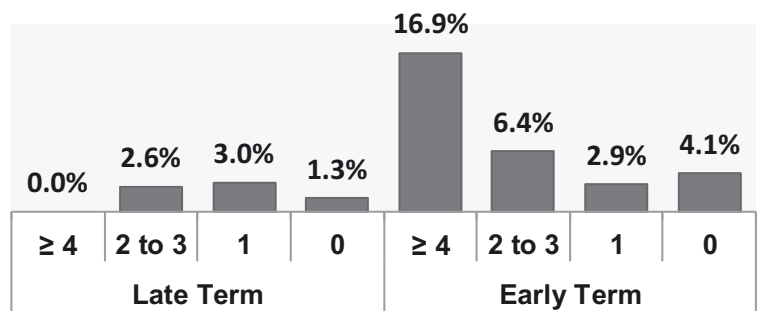

Figure 1: The number of prior caesarean sections and neonatal outcomes. 
section was performed beyond 39 completed weeks. A significant drop in the NICU admission was also demonstrated when elective caesarean sections were performed after 39 completed weeks.

Majority of the mothers who had elective LSCS in both groups had repeat caesarean sections and our data suggests that women with higher order caesarean sections were sectioned at earlier gestation in their current pregnancies. We thus observed that while about half of those who had one previous LSCS were done after 39 weeks, only $1 \%$ of those who had four or more previous caesarean sections were performed at full term.

The international healthcare community has long debated about an ideal but safe rate for caesarean section over the years. Despite this, the last two decades have witnessed an increasing rise in the rate of both primary and repeat cesarean sections in both developed and developing countries $[1,2]$. This trend poses major health concerns because of its associated maternal and neonatal morbidity and mortality [3-5]. The detrimental effects of performing elective CS prior to 39 weeks of gestation on neonatal respiratory outcome have been extensively studied in many parts of the world. Previous studies have shown that the risk of neonatal respiratory morbidity was about 10 times more in neonates born by CS [13-15]. According to a Maleda and colleagues [14], the risk of respiratory morbidity was 6.3 times more likely to occur in early-term neonates delivered by elective cesarean section in contrast to a 2.4 times more likelihood in late-term neonates delivered by elective cesarean delivery. In our study, the need for some form of respiratory support remained even after adjustment for other factors. RANZOG statement of timing of elective CS quotes "when compared with either planned or achieved vaginal birth, elective caesarean birth is associated with a 2.1-6.8-fold increase in the risk of these respiratory morbidities in the near term neonate" [9]. Most of the international bodies including Royal College of Obstetricians and Gynecologists (RCOG) and American College of Obstetrician and Gynecologists (ACOG) have recommended performing elective CS after 39 completed weeks of gestation.

Despite the global evidence-based recommendation to schedule an elective cesarean section after 39 completed weeks, strict adherence to this policy often fails due to multiple complex factors. This includes a large patient load which exceeds the available resources, fear of patient going into labor prior to the assigned date (with associated maternal and neonatal risks of out- of- hours CS), practice of scheduling potentially complicated cases (for example, higher order caesarean sections) during the hours when personnel with advanced skills are easily available. This argument points to the fact that when balancing known neonatal benefits against potential maternal risks, obstetric teams tend to prioritize the latter over the former and tend to schedule elective CS before 39 completed weeks.

Our hospital is one of the largest obstetric units in the Middle East, catering for a large volume of multicultural obstetric population with complex needs. The complexities involved sometimes necessitate scheduling elective CS prior to 39 completed weeks on multiple occasions. This could be the attributed to the fear of patient going into spontaneous labor before the assigned date which significantly increases maternal and neonatal risks and may necessitate performing complicated cases outside the regular operating hours. By scheduling higher order caesarean sections at earlier gestation to eliminate the small risk $(<10 \%)$ of intrapartum caesarean sections, the obstetricians are playing a major role in increasing the magnitude of the neonatal respiratory morbidity. Therefore, we strongly suggest revising our current practice of scheduling higher order caesarean section at earlier gestational age. Equally important, is the need to reduce the number of primary caesarean sections and repeat caesarean sections by interventions such as encouraging trial of labor after caesarean sections, encouraging vaginal breech delivery and external cephalic version and discouraging caesarean section on maternal request.

\section{Strengths and limitations}

To the best of our knowledge, this is the first study on this subject in this country.

This study had a large sample size and we minimized for selection bias by examining all available births for the study period. In addition, we also minimized for selection bias by removing complicated pregnancies and pregnancies with a prior history of antenatal steroid use before birth. With regards to the practice of antenatal corticosteroids for early term planned CS, there is conflicting advice from international organizations. RCOG recommends administration of antenatal corticosteroids to all pregnant women for whom an elective caesarean section is planned prior to $38+6$ weeks of gestation [8]. RANZOG supports this by stating “... Where delivery by caesarean section (without prior labor) is planned before 39 weeks gestation, consideration should be given to the administration of corticosteroids to reduce respiratory morbidity in the newborn" [9]. However, other international bodies such as the Society for Maternal-Fetal Medicine (SMFM) do not advocate the routine use of steroids before early term (ET) elective CS $[16,17]$. In our obstetric unit, we do not routinely 
give steroids to mothers who undergo elective LSCS before 39 completed weeks. We therefore do not know if the observed adverse respiratory morbidities would have had better outcomes as reported in other studies [18].

Despite the study strengths, our study is not without limitations. Potential issues from using routine health data include misclassification bias, missing data from incomplete and variable documentation including measurement bias [19]. This was a retrospective data analysis and variation within patient notes regarding gestational age cannot be ruled out.

\section{Conclusions}

We examined respiratory morbidities and neonatal outcomes in term livebirths according to various gestational age in our population showing a higher incidence of adverse outcomes in the early term CS newborns compared to late term newborns. We strongly advocate adherence to the existing guidelines of performing LSCS after 39 weeks at national and global levels to mitigate the short term and long term neonatal respiratory morbidity. We hope the results would help obstetricians to optimally schedule elective CS for the best maternal and neonatal outcomes.

Research funding: The study is funded by Qatar National Research Fund (QNRF) and sponsored by the Medical Research Centre, Hamad Medical Corporation.

Author contributions: All authors have accepted responsibility for the entire content of this manuscript and approved its submission.

Competing interests: Authors state no conflict of interest. Informed consent: The study received a waiver of written consent.

Ethical approval: The study was approved by the Hamad Medical Corporation Institutional Review Board.

\section{References}

1. Ye J, Betran AP, Guerrero Vela M, Souza JP, Zhang J. Searching for the optimal rate of medically necessary cesarean delivery. Birth 2014;41:237-44.

2. Vogel JP, Betrán AP, Vindevoghel N, Souza JP, Torloni MR, Zhang J, et al. Use of the robson classification to assess caesarean section trends in 21 countries: a secondary analysis of two WHO multicountry surveys. Lancet Global Health 2015;3:e260-70.
3. Keag OE, Norman JE, Stock SJ. Long-term risks and benefits associated with cesarean delivery for mother, baby, and subsequent pregnancies: systematic review and meta-analysis. PLoS Med 2018;15:e1002494.

4. Morrison JJ, Rennie JM, Milton PJ. Neonatal respiratory morbidity and mode of delivery at term: influence of timing of elective caesarean section. BJOG An Int J Obstet Gynaecol 1995;102: 101-6.

5. Hansen AK, Wisborg K, Uldbjerg N, Henriksen TB. Risk of respiratory morbidity in term infants delivered by elective caesarean section: cohort study. BMJ 2008;336:85-7.

6. Wax JR, Herson V, Carignan E, Mather J, Ingardia CJ. Contribution of elective delivery to severe respiratory distress at term. Am J Perinatol 2002;19:081-6.

7. Zanardo V, Alphonse Simbi K, Vedovato S, Trevisanuto D. The influence of timing of elective cesarean section on neonatal resuscitation risk. Pediatr Crit Care Med 2004;5:566-70.

8. National Institute for Health and Care Excellence (NICE). Caesarean Section guideline. 2011.

9. The Royal Australian and New Zealand College of Obstetricians and Gynaecologists (RANZCOG). Timing of elective caesarean section at term; 2006, Report No.: C-Obs 23.

10. ACOG. Committee opinion no. 561. Obstet Gynecol 2013;121: 911-5.

11. Guglani L, Lakshminrusimha S, Ryan RM. Transient tachypnea of the newborn. Pediatr Rev 2008;29:e59-65.

12. Martin R. Pathophysiology, clinical manifestations, and diagnosis of respiratory distress syndrome in the newborn UpToDate [Internet]; 2020. Available from: https://www. uptodate.com/contents/pathophysiology-clinicalmanifestations-and-diagnosis-of-respiratory-distresssyndrome-in-the-newborn [Cited 20 Dec 2020].

13. MacDorman MF, Declercq E, Menacker F, Malloy MH. Infant and neonatal mortality for primary cesarean and vaginal births to women with "no indicated risk," United States, 1998-2001 birth cohorts. Birth 2006;33:175-82.

14. Tefera M, Assefa N, Mengistie B, Abrham A, Teji K, Worku T. Elective cesarean section on term pregnancies has a high risk for neonatal respiratory morbidity in developed countries: a systematic review and meta-analysis. Front Pediatr 2020;8:286.

15. Many A, Helpman L, Vilnai Y, Kupferminc MJ, Lessing JB, Dollberg S. Neonatal respiratory morbidity after elective cesarean section. J Matern Fetal Neonatal Med 2006;19:75-8.

16. Society for Maternal-Fetal Medicine Publications Committee S. Implementation of the use of antenatal corticosteroids in the late preterm birth period in women at risk for preterm delivery. Am J Obstet Gynecol 2016;215:B13-5.

17. ACOG. Committee opinion no. 713. Obstet Gynecol 2017;130:e102-9.

18. Saccone G, Berghella V. Antenatal corticosteroids for maturity of term or near term fetuses: systematic review and meta-analysis of randomized controlled trials. BMJ 2016;355:i5044.

19. Benchimol El, Smeeth L, Guttmann A, Harron K, Moher D, Petersen I, et al. The reporting of studies conducted using observational routinely-collected health data (RECORD) statement. PLoS Med 2015;12:e1001885. 